\title{
Conformational Adaptation of ApolipoproteinA-I To Discretely Sized Phospholipid Complexes
}

\author{
Shaila Bhat $\%$, Mary G Sorci-Thomas ${ }^{\star}, \% 1$, Rubina Tuladhar ${ }^{*}$, Michael P. Samuel ${ }^{*}$, and Michael \\ J. Thomas \\ *Department of Biochemistry, Wake Forest University Medical Center, 391 Technology Way, Building Al, \\ Winston-Salem, NC 27101 \\ \%Department of Pathology, Center for Lipid Science, Wake Forest University Medical Center, 391 \\ Technology Way, Building A1, Winston-Salem, NC 27101
}

\section{Abstract}

The conformational constraints for apoA-I bound to recombinant phospholipid complexes (rHDL) were attained from a combination of chemical cross-linking and mass spectrometry. Molecular distances were then used to refine models of lipid-bound apoA-I on both 80 and $96 \AA$ diameter rHDL particles. To obtain molecular constraints on the protein bound to phospholipid complexes, three different lysine-selective homo-bifunctional cross-linkers with increasing spacer arm lengths (i.e., 7.7, 12.0, and $16.1 \AA$ ) were reacted with purified, homogeneous recombinant 1-palmitoyl-2-oleoylsn-glycerol-3-phosphocholine (POPC) apoA-I rHDL complexes of each diameter. Cross-linked dimeric apoA-I products were separated from monomeric apoprotein using 12\% SDS PAGE, then subjected to in-gel trypsin digest, and identified by MS/MS sequencing. These studies aid in the refinement of our previously published molecular model of 2 apoA-I molecules bound to $\sim 150$ molecules of POPC and suggest that the protein hydrophobic interactions at the $\mathrm{N}$ - and $\mathrm{C}$-terminal domains decrease as the number of phospholipid molecules or "lipidation state" of apoA-I increases. Thus, it appears that these incremental changes in the interaction between the $\mathrm{N}$-and $\mathrm{C}$-terminal ends of apoA-I stabilize its tertiary conformation in the lipid-free state as well as allowing it to unfold and sequester discrete amounts of phospholipid molecules.

Apolipoprotein A-I (apoA-I) is a $28 \mathrm{kDa}$ protein synthesized by the liver and intestine and is responsible for modulating the formation, metabolism, and catabolism of high density lipoprotein cholesterol. HDL has been known for decades to be a negative risk factor for predicting the development of coronary artery disease in humans, but the specific mechanism (s) responsible for its protective role in cholesterol metabolism continues to be studied and elucidated (1-3).

ApoA-I shares a number of similarities to other members of the apoprotein super gene family, as well as possessing a unique set of properties related to its unique role in lipid metabolism (4). Totally soluble in aqueous solution, apoA-I monomers exist in a lipid-free state, but also avidly bind to each other, as well as lipid surfaces (5). Although apoA-I readily binds lipid surfaces, the formation of small apoA-I containing phospholipid and cholesterol particles, an important step in HDL metabolism, does not occur to a significant extent in the absence of the ABCA1 transporter. During the formation of "nascent" HDL, monomers of lipid-free apoA-I bind to ABCA1 which adds phospholipid and cholesterol to yield a particle containing two

1To whom correspondence should be addressed: Dept. of Pathology, Section on Lipid Sciences, Wake Forest University Health Sciences, Medical Center Blvd., Winston-Salem, NC 27157. Tel.: 336-716-2147; Fax 336-716-6279; E-mail:msthomas@wfubmc.edu. 
molecules of apoA-I $(3,6,7)$. In this form, the conformation of apoA-I is an excellent coactivator for the plasma enzyme lecithin:cholesterol acyl transferase (LCAT) that rapidly converts apoA-I containing lipid particles to mature or spherical HDL with a core of cholesterol ester. If any part of this process is interrupted or impaired, the small apoA-I particles are rapidly removed from circulation by the kidney (6). Studies of human deficiencies have shown that if either ABCA1 or LCAT are inactive, plasma concentrations of HDL apoA-I are very low due to the rapid removal of lipid-poor HDL apoA-I from circulation $(8,9)$. Additionally, recycling of HDL apoA-I may occur through delipidation of HDL particles by SR-B1 or through remodeling of mature HDL particles $(7,10,11)$.

Structurally, apoA-I contains a 1-43 residue N-terminal globular region, referred to as a $\mathrm{G}^{*}$ amphipathic alpha helix (12) followed by 10 amphipathic alpha helices, of the $\mathrm{Y}$ and A classes, which comprise the remaining $80 \%$ of the protein. The $\mathrm{N}$-terminus or flexible domain contains three 11-mer tandem repeats (residues 11-21, 22-32, and 33-43, termed helices G1, G2 and G3) (13). It is generally believed that the N-terminus is important for the stabilization of the lipid-free apoA-I conformation (14), while the C-terminus (\#209-243) is highly hydrophobic, composed of Y-class amphipathic helices and important for lipid binding $(12,15,16)$. The structure of the $\mathrm{N}$ - and C-terminal regions have been studied extensively and shown to be mainly non-helical in the lipid-free state but take on some alpha-helical character upon lipid binding (17-21). Although the $\mathrm{N}$-and C-terminal domains both appear to be involved in maintaining the stability of the lipid-free conformation and binding to lipids, these domains also appear to have distinct functions $(5,22,23)$.

Functionally, the C-terminus has been implicated in the initiation of binding to ABCA1 and/ or lipid surfaces $(18,24)$, while the N-terminus has been linked to LCAT activation and HDL maturation (25). Studies show that the $\Delta 43$ mutant form of apoA-I exhibits similar lipid binding ability to full length apoA-I (21). Interestingly, when both the $\mathrm{N}$ - and $\mathrm{C}$-terminal regions are removed, both lipid binding and ABCA1-mediated lipid efflux are similar to full-length apoAI $(18,24,26)$. These results suggest that in the presence of lipid the central region of apoA-I comprising residues 44-186 can exist as a stable independently folded fragment, however, removal of one or the other terminal ends disrupts the ability of the lipid-free protein to stabilize its structure in solution, possibly through interaction with each other $(18,24)$.

Interestingly, deletion of the 1-43 residue region of apoA-I also has little effect on the overall size of 95-105 A diameter recombinant phospholipid particles generated with lipid-free apoAI (13). Based on these observations it has been hypothesized that the 1-43 flexible domain is either unassociated with the phospholipid particle and exists as either a random or helical coiled-coil, or associates with the face of the phospholipid particle as either an amphipathic alpha helix or $\beta$-strand (13). Indeed, recent studies show that the $\mathrm{N}$-terminus contains a short segment of $\beta$-strand structure which may be similar in length to a similar stretch observed in the C-terminus $(19,20)$ creating a potential region for intra-or inter-domain interaction.

To date, phospholipid containing apoA-I particles have not yielded crystals of the quality needed to solve the structure of lipid bound apoA-I by X-ray crystallography. However, the $\mathrm{X}$-ray crystal structure of full length lipid-free apoA-I has been recently reported by Ajees et al. (27). These investigators were the first to show that full-length lipid-free apoA-I exists in a four-helix bundle. This structure differs significantly from the X-ray crystal structure of lipidfree mutant apoA-I lacking amino acids \#1-43 ( $\Delta 43$ apoA-I), reported by Borhani et al. in 1997 (14). Lipid-free $\Delta 43$ apoA-I crystallized in a "horseshoe" or "belt" shaped structure assuming an anti-parallel orientation in which helix 5 of one molecule was "in register" with helix 5 of a second apoA-I molecule. This structure triggered renewed interest in solving the conformation of lipid-bound apoA-I shifting the paradigm away from a "picket-fence" to a 
"double belt" model (28). Since then, numerous studies have provided additional support for the double-belt model of lipid-bound apoA-I (29).

Most recent to the arsenal of methods employed to solve the lipid-bound structure of apoA-I, chemical cross-linking combined with mass spectrometry (CCL/MS), provides a lowerresolution picture of protein structure when compared to X-ray crystallography or NMR spectrometry. However, CCL/MS can provide valuable information on protein structure when $\mathrm{X}$-ray and NMR procedures cannot be used, e.g., due to poor crystal quality or particle aggregation. An important validation of CCL/MS for structure analyses was demonstrated by Young et al. (30) in which distance constraints for fibroblast growth factor-2 were compared and found to agree well with results derived from X-ray crystallography.

To date, two independent groups have used CCL/MS to study the lipid-bound conformation of apoA-I (31-33). A comparison of these two models agree quite well with each other except for cross-links that did not fit the established LL5/5 extended belt conformation (34). In the LL5/5 conformation two molecules of apo A-I are antiparallel and exist with helix 5 in registry with helix 5 of the second molecule of apoA-I. In one model, cross-links not conforming to the LL5/5 model were assigned to a second conformation in which two molecules of apoA-I are antiparallel and folded in a helix 5 to helix 2 registry (LL5/2). This alignment is believed to allow the central domain corresponding to helices 5-7 (amino acids 121-186) to loop out to accommodate smaller particle diameters $(33,35)$. In the other model of lipid-bound apoAI, cross-links that did not fit the LL5/5 conformation were hypothesized to fit a conformation in which both the $\mathrm{N}$ - and $\mathrm{C}$-termini of the lipidated apoA-I formed a hairpin structure which could then fold back and interact with themselves (32). In the current studies, this model of lipid-bound apoA-I was re-examined and probed using cross-linkers of different lengths on two different sizes of recombinant particles. These results provide additional support for a model which suggests protein to protein interaction between the $\mathrm{N}$ - and $\mathrm{C}$-terminal regions of 2 apoA-I molecules bound to 96 and $80 \AA$ diameter phospholipid particles.

\section{EXPERIMENTAL PROCEDURES}

\section{Materials}

Disuccinimidyl glutarate (DSG), dithiobis(succinimidylpropionate) (DSP) and ethylene glycol bis(succinimidylsuccinate) (EGS) were procured from Pierce. POPC and $\mathrm{Me}_{2} \mathrm{SO}$ were purchased from Sigma. Sequencing grade modified trypsin and restriction enzymes were from Promega. RapiGest SFTM was obtained from Waters Inc. Formic acid was purchased from Aldrich. Sodium desoxycholate, potassium chloride, optima grade methanol, chloroform, acetonitrile, and glacial acetic acid were obtained from Fisher Scientific. Mark 12 molecular weight standards and Simply Blue ${ }^{\mathrm{TM}}$ Safestain were obtained from Invitrogen. Ultrafree-15 centrifugal and Biomax ${ }^{\mathrm{TM}} 10 \mathrm{~K}$ membranes were from Millipore Corp.

\section{Preparation and Purification of 96 and $80 \AA$ Human ApoA-I Containing rHDL}

Human apoA-I was purified from plasma using standard procedures (36) and its purity was checked by mass spectrometry. The preparation of $96 \AA$ rHDL was carried out as described previously (32). The preparation of $80 \AA \mathrm{rHDL}$ was carried out using 1 mole of apoA-I to 10 moles of POPC. Briefly, POPC dissolved in chloroform was dried under vacuum, then mixed with sodium desoxycholate at a ratio of 2 cholate : 1 POPC and vortexed for an hour (32). After extensive dialysis, the crude $80 \AA$ rHDL was subjected to ultracentrifugation to remove lipidfree apoA-I from lipidated apoA-I. First the density of the crude mixture was adjusted to 1.29 $\mathrm{g} / \mathrm{ml}$ with $\mathrm{KBr}$ then a $1.26 \mathrm{~g} / \mathrm{ml} \mathrm{KBr}$ solution was overlayered and the tube spun at $50 \mathrm{~K}$ for $22 \mathrm{~h}$ at $15{ }^{\circ} \mathrm{C}$. After centrifugation the top fraction was dialyzed and then analyzed by nondenaturing gradient gel electrophoresis to check the homogeneity and size of the rHDL (32). 


\section{Cross-linking of 96 and $80 \AA ̊$ Lipid-bound ApoA-I}

The cross-linkers DSG, DSP or EGS were dissolved in $\mathrm{Me}_{2} \mathrm{SO}$ at a final concentration of 5 $\mu \mathrm{g} / \mu \mathrm{l}$ and used within $5 \mathrm{~min}$ of preparation. For lipid-bound 96 and $80 \AA$ A rHDL, the crosslinkers were added at molar ratios of 2:1, 20:1 and 200:1 with a final rHDL apoA-I particle concentration of $0.4 \mu \mathrm{g} / \mu \mathrm{l}$ in a $10 \mathrm{mM}$ sodium phosphate $\mathrm{pH} 7.4$ (32). After adding the crosslinker, the reaction was incubated for $5 \mathrm{~min}$ at $37^{\circ} \mathrm{C}$ then quenched by adding $1 \mathrm{M}$ Tris $\mathrm{pH} 7.4$ to a final concentration of $50 \mathrm{mM}$. The samples were dialyzed against $10 \mathrm{mM}$ ammonium bicarbonate $\mathrm{pH} 7.4$ at $4{ }^{\circ} \mathrm{C}$ to remove excess cross-linker. All samples were stored at $-20{ }^{\circ} \mathrm{C}$ until further processed.

\section{Non-Reducing 12\% SDS PAGE and In-gel Trypsin Digest}

The cross-linked apoA-I rHDL products were separated on 12\% SDS-PAGE under nonreducing conditions (32) and visualized using SimplyBlue. Lipid-free human apoA-I and Q109C apoA-I were included on the gel as markers for the migration of monomer and the D80dimer, respectively. Product bands were excised from the gel dehydrated with rehydrated with cold, freshly prepared trypsin solution containing $20 \mathrm{ng} / \mu \mathrm{l}$ in $10 \mathrm{mM}$ ammonium bicarbonate, $\mathrm{pH} 7.8,0.1 \%$ (w/v) RapiGest $\mathrm{SF}$ and $1 \mathrm{mM} \mathrm{CaCl}_{2}$, as previously described (32). The final trypsin to apoA-I mass ratio was $1: 20$. After incubating on ice for $10 \mathrm{~min}$. the digests were then incubated for $18 \mathrm{~h}$ at $37^{\circ} \mathrm{C}$.

\section{Peptide Isolation and ES/Q-TOF Mass Spectrometry}

Following digestion of the cross-linked apoA-I, the resulting peptides were extracted from the gel slices using $200 \mu \mathrm{l}$ of extraction buffer (acetonitrile/formic acid/water, v/v/v, 50:5:45). After incubating the slices for $10 \mathrm{~min}$ in this buffer the peptides were transferred to a fresh tube. A second extraction was then carried out and the combined aliquots acidified to an $\mathrm{HCl}$ : apoA-I ratio of 1:10 (v/v) using a $500 \mathrm{mM} \mathrm{HCl}$. The solution was incubated for $35 \mathrm{~min}$ at $37^{\circ}$ $\mathrm{C}$ then centrifuged for $10 \mathrm{~min}$ at $13,000 \mathrm{rpm}$. The supernatant was transferred to a fresh tube before processing for mass spectrometry.

Survey scans were performed on each sample and candidate peptides were picked for sequencing. Peptides were sequenced using a Waters Q-TOF API-US mass spectrometer equipped with a Waters CapLC as described previously (32) using the Mass-Lynx ${ }^{\mathrm{TM}} 4.0$ software. Peptides were loaded onto a PLRP-S trapping column having a $3 \mu$ particle size, 100 $\AA$ pore size, $0.5 \mathrm{mM}$ diameter and $2.0 \mathrm{mM}$ length. Peptides were first loaded onto the column in water/acetonitrile/formic acid (97:3:0.2) at $500 \mathrm{nl} / \mathrm{min}$ then eluted using a gradient starting with solvent A ( $25 \mathrm{mM}$ formic acid in $97 \%$ water and 3\% acetonitrile). This was followed by $2 \%$ solvent B ( $25 \mathrm{mM}$ formic acid in 3\% water $97 \%$ acetonitrile). The gradient profile was set at $2 \%$ solvent B for $3 \mathrm{~min}, 40 \% \mathrm{~B}$ at $90 \mathrm{~min}, 80 \% \mathrm{~B}$ at $95 \mathrm{~min}, 2 \% \mathrm{~B}$ at $100 \mathrm{~min}$ and $2 \% \mathrm{~B}$ at 130 min with a flow rate of $470 \mathrm{~nL}$ to the column. Positive ion electrospray spectra were recorded in the continuum mode using a scan window from 300 to $1500 \mathrm{~m} / \mathrm{z}$ and an accumulation time of $2 \mathrm{~s}$ using a charge state selective collision energy and a $2 \mathrm{~s}$ accumulation time, product ion spectra were acquired in the continuum mode from 50 to $1800 \mathrm{~m} / \mathrm{z}$. The source temperature was $80^{\circ} \mathrm{C}$. The cone and capillary voltages were $45 \mathrm{~V}$ and $3.5 \mathrm{kV}$, respectively.

\section{Cysteine-Containing ApoA-I Homo- and Hetero-Dimer Mobility Studies}

The cysteine containing mutant apoA-I proteins used in the homodimer migration studies were constructed and purified as previously described $(32,37,38)$. Each of the purified cysteinecontaining apoA-I mutants were analyzed by mass spectrometry (39) to confirm that their experimental and theoretical molecular weights were the same. From previous studies it was noted that cysteine homodimers from Q109C and Q132C apoA-I migrated at $\sim>66 \mathrm{kDa}(32)$, which are referred to as Dimer 2 (D2) while cysteine homodimers from Q84C and S231C 
apoA-I were found to migrate around the expected $\sim 56 \mathrm{kDa}$ (MW of apoA-I is $\sim 28,000$ ) and are referred to as Dimer 1 (D1). For the migration studies both reduced lipid-free Q132C apoAI and S231C apoA-I were radiolabeled with ${ }^{125}$ I using the iodine monochloride method (40) and mixture of the two were used as molecular size markers for non-reducing SDS PAGE detection of the D1 and D2 migration, respectively. In a typical experiment, $1 \times 10^{5} \mathrm{cpm}$ representing trace amounts of the $\mathrm{I}^{125}$ labeled Q132C apoA-I (or I $\mathrm{I}^{125}$ labeled S231C) apoA-I was added to $3 \mu \mathrm{g}$ of unlabeled cystiene apoA-I mutant proteins. The mixtures was then adjusted to $50 \mathrm{mM}$ DTT and incubated for $30 \mathrm{~min}$ on ice. Formation of cysteine-containing apoA-I homo- and heterodimers was assisted by the removal of DTT via dialysis against 10 $\mathrm{mM}$ ammonium bicarbonate $\mathrm{pH}$ 7.4. The migration of radiolabeled apoA-I hetero-and homodimers were characterized on non-reducing $12 \%$ SDS PAGE followed by radioactive imaging on a Typhoon 8600 Variable Mode Imager from Molecular Dynamics and ImageQuant 5.2 software.

\section{Molecular Modeling of Lipid-Free and Lipid-Bound ApoA-I}

Four basic pieces of information were needed in order to model the conformation of the two apoA-I molecules on POPC rHDL particles. The first step was to orient the positions of the individual apoA-I molecules that were cross-linked relative to each other. To do this we used the maximum distance of $\mathrm{C}_{\alpha}$-Lysine-(cross-linker)- $\mathrm{C}_{\alpha}$-Lysine calculated for each of the crosslinkers; $22.3 \AA$, $26.6 \AA$, and $30.7 \AA$ for DSG, DSP and EGS, respectively. Next, the apoA-I structure was constructed from coordinates from lipid-free $\Delta 43$-apoA-I (14) that were joined with the 1-43 amino acids reported for the crystal structure of full-length lipid-free apoA-I (27). Thirdly, apoA-I was bent only at the proline sites between the amphipathic segments of apoA-I. Lastly, the two molecules of apoA-I were arranged antiparallel to one another. Tools available in Swiss-PdbViewer vs OSX 3.9b1.01 (http://www.expasy.org/spdbv/) were used to optimize the conformations while pdb files were manipulated using PyMOL

(http:/www.pymol.org). Swiss-PdbViewer, PyMOL along with VMD for Mac OSX, version 1.8.4 (41) were used to generate the molecular figures shown in the manuscript.

\section{RESULTS}

\section{Characterization and Cross-linking of 96 and $80 \AA \AA$ rHDL Containing ApoA-I}

Purified $96 \AA$ diameter rHDL particles were found to contain an average of 150 molecules of POPC to 2 molecules of apoA-I, while purified $80 \AA$ Aiameter rHDL contained approximately 54 molecules of POPC to 2 molecules of apoA-I (see supplemental Figure 1 for 4-30\% ND GGE used for sizing). Once purified, all rHDL were treated with the amine specific crosslinkers, DSG, DSP and EGS, having spacer arm lengths of 7.7, 12.0 and 16.1 A, respectively (see supplemental Figure 2 for chemical structure of these thio-cleavable cross-linkers). Each cross-linker was used at several different molar ratios relative to rHDL apoA-I mass in order to obtain distance constraints between the two molecules of apoA-I residing on different sized phospholipid containing particles.

Cross-linked products obtained from these reactions were separated by $12 \%$ SDS PAGE as shown in Figure 1, for $96 \AA$ diameter (Panels A, C, and E) and for $80 \AA$ diameter (Panels B, D, and F) POPC rHDL apoA-I reacted with DSG (Panels A and B), DSP (Panels C and D) and EGS (Panels E and F) at 3 different molar ratios of cross-linker to protein. Striking similarities were seen between the distribution of apoA-I cross-linked products when the 96 and $80 \AA$ POPC rHDL were compared as well among the different cross-linkers used. Although less consistent among all the conditions shown in Figure 1, cross-linked product bands were also seen above and between D1 and D2. Product bands between D1 and D2 mostly likely represent cross-linked products at different sites along the primary sequence of the two apoA-I protein molecules, while cross-links seen above, most likely reflect higher order oligomerization. A 
more detailed analysis addressing specific cross-linked regions leading to aberrant SDS PAGE migration is presented in the next section. Interestingly, product distribution was also similar among the different cross-linkers used (EGS, DSP, and DSG). Of particular significance, was the observation that at low cross-linker to apoA-I molar ratios (1:10) two distinct sized dimer products were formed. These cross-linked dimers (referred to as D1 and D2) have been previously investigated (32) and have been shown to represent distinct intermolecular covalent attachments within apoA-I. The different SDS PAGE mobility for these two similar apoA-I dimers has been previously reported (32) and does not represent differences in amino acid number or composition, rather, MS/MS sequencing of cross-linked apoA-I peptides showed that apoA-I dimer attachment within the central region was related to the aberrant SDS PAGE migration (32).

\section{Electrophoretic Migration of Homo- and Hetero-Dimers of Cysteine-Containing ApoA-I}

The altered SDS PAGE migration of apoA-I dimers as a function of covalent site attachment was investigated using homo-and hetero-dimers formed from the oxidation of cysteine containing apoA-I mutants. Figure 2 Panel A, shows an SDS PAGE gel run under non-reducing conditions and stained for protein mass. In this panel, each of the single cysteine substitution mutants of apoA-I show a monomer band at the expected 28,000 Da, as well as a dimer band running at approximately 55,000 Da, except for Q109C and Q132C apoA-I (Panel A, Lanes 4 and 5). Since, cysteine containing apoA-I monomers will oxidize to form homodimers in the absence of reducing agents, such as DTT or $\beta$-mercaptoethanol, each protein shows both the monomer and dimer form for each cysteine containing mutant apoA-I protein. Of particular interest, Q109C and Q132C apoA-I show the largest deviation from the calculated dimer molecular weight of $\sim 56,000 \mathrm{Da}$, while the remaining apoA-I cysteine substitution mutations show migration closer to the theoretical molecular weight. All cysteine containing apoA-I mutants shown in panel A were subjected to mass spectrometry, in momoner (+DTT) and dimer (-DTT) form, as previously described (39) to confirm theoretical to actual molecular weights.

It is well known that the interaction of SDS with a wide variety of proteins yields a rod-like particle in which the length varies uniquely with the molecular weight of the protein (42). However, deviations between theoretical and experimental molecular weights as determined by SDS PAGE have been shown for a number of proteins and may result from the resistance of certain proteins to SDS unfolding The aberrant migrating apoA-I dimer most likely represent a conformation that is either structurally rigid or highly kinetically stable (43). Figure 2 Panel $\mathrm{B}$, shows a hypothetical model illustrating the linkage and conformation for a homodimer of S231C apoA-I, (Dimer 1), and a homodimer of Q132C apoA-I, referred to as Dimer 2. A slower migration on SDS PAGE for Dimer 2 can be imagined if the less "linearized", D2 encounters interference migrating through the polyacrylamide pores, resulting in a larger than actual molecular size compared to D1 which is more linearized and can migrate as a rod through the pores.

Additional studies were conducted to investigate the SDS PAGE migration of heterodimers of cysteine containing apoA-I proteins. The migration of heterodimers containing radiolabeled Q132C apoA-I and combinations of different cysteine containing apoA-I mutants are shown in Figure 2, Panel C. In Panel C, top and bottom, Lanes 1 and 6 show a mixture of the radiolabeled homodimers of S231C and Q132C apoA-I, which were mixed for mobility reference on SDS PAGE. In Panel C, top, radiolabeled Q132C apoA-I was mixed with cold cysteine containing apoA-I, reduced with DTT then dialyzed and run on non-reducing SDS PAGE. These results show that the presence of heterodimers with the covalent attachment of cysteine 132 to cysteines at either position 84 (Lane 3) or 231 (Lane 5) resulted in migration with the D2. In Panel C, bottom, radiolabeled S231C apoA-I was mixed with cold cysteine containing apoA-I as above. These results show that the heterodimer with covalent attachment 
of cysteine 231 at either position 109 (Lane 7) or 132 (Lane 8) also resulted in migration at D2. These results suggest that a single covalent attachment within the central domain of apoAI, whether it involves two molecules attached at the same site (homodimer), or one molecule attached within the central domain and one site at a $\mathrm{N}$-or $\mathrm{C}$-terminal domain (heterodimer) retards the SDS PAGE migration. While heterodimers containing radiolabeled S231C apoAI showed a mixture of both Dimer I and Dimer 2. The molecular basis explaining these results may relate to the size or shape of the dimer or to the kinetic stability and inability of the dimer to be "relaxed" by treatment with SDS.

\section{In-gel Trypsin Digest of Cross-linked ApoA-I and Mass Spectrometry}

Cross-linked monomeric and dimeric apoA-I products were excised and digested with trypsin then the extracted peptides analyzed by ES-MS/MS. After mass correction a peptide list was generated from the survey scan and compared to a theoretical list of all possible peptide crosslinks. These theoretical lists were constructed for each chemical cross-linker. After mass correction candidate peptides were assumed to have masses that were within $\pm 10 \mathrm{ppm}$ of the calculated mass. After picking candidate peptides from the survey scans, samples were reanalyzed and candidate peptides sequenced by MS/MS. Survey scans yielded a total of 41,30 , and 53 candidate cross-linked peptides from $96 \AA$ POPC rHDL apoA-I treated with DSG, DSP and EGS, respectively, out of which 41, 27 and 46 provided sequence information. From these sequenced peptides 29, 19 and 45 proved to be uncross-linked peptides.

Table 1 shows all of the cross-links identified from a 96^ POPC rHDL apoA-I with DSG, DSP and EGS. Three of the four intermolecular and four of the seven intramolecular cross-links were found with DSG. The only cross-link found from treatment with EGS was intramolecular between lysines 133 and 140 (and also found with DSG, and DSP). Figure 3 Panel A shows the MS/MS sequence for one intermolecular DSG cross-link between lysines 12 and 94 isolated from a D1 product from $96 \AA$ POPC rHDL.

However, unlike the $96 \AA$ POPC rHDL, the $80 \AA$ diameter rHDL yielded a total of 13,8 and 5 candidate cross-linked peptides after treatment with DSG, DSP and EGS respectively, out of which only 10, 5 and 2 peptides for each of the cross-linkers, respectively could be sequenced. Of these candidate sequences 6,3 , and 1 proved to be uncross-linked peptides and only 4, 2 and 1 for DSG, DSP and EGS respectively, were identified as valid cross-links via sequencing. Out of all the potential cross-links, only two ions that did not fragment even after the collision energy was raised to 30, while the ion 738.03 ion for DSG cross-linked $80 \AA$ POPC rHDL had a mixture of two different charge states and was unable to be sequenced. Table 2 shows all the intermolecular and one intramolecular cross-links identified from 80A POPC rHDL apoA-I. Figure 3 Panel B, shows the MS/MS sequence of an intermolecular DSG cross-link between lysines $\alpha-\mathrm{NH} 2$ and 118 from the $80 \AA$ POPC rHDL. Here the product ion spectrum for the 2190.0742 Da fragment was obtained from a D1 product. The MS/MS spectrum was obtained from the triply charged ion $\mathrm{m} / \mathrm{z} 731.01$ and the sequence of the peptide is shown as single letter abbreviations.

\section{Molecular Models for ApoA-I Folding on 96 and $80 \AA \AA$ rHDL Complexes}

For apoA-I bound to $96 \AA$ rHDL particles, Figure 4 Panels A, B and C shows the positioning for each of the two lipid-bound molecules based on all Lys-Lys intermolecular cross-links listed in Table 1. Most specifically in Figure 4 Panel D, the resulting conformation shown in Panels A-C, results from an interlocking network of intramolecular cross-links (yellow lines)

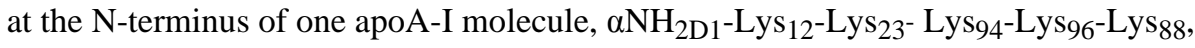
connected through a intermolecular cross-link (red lines) at Lys 12 connected to Lys 182 of the second apoA-I molecule. Additionally, a second intermolecular cross-link between the two antiparallel molecules at $\mathrm{Lys}_{40}$ and $\mathrm{Lys}_{239}$ demonstrated that considerable lengths of the $\mathrm{N}$ - 
terminal and C-terminal ends of opposing strands are closely associated (Figure 4, Panel D). Overall, these cross-linked sites indicate that the N-terminal end of each apoA-I molecules folds back and associates with itself and that the C-terminal ends of one apoA-I associates closely with the N-terminal end of the second apoA-I. Intermolecular cross-links between Lys $_{118}$ to Lys ${ }_{140}$ show that the "centers" of the antiparallell apoA-I molecules are also closely associated with repeats 5 of each apoA-I molecule centered over one another, consistent with studies on helix registry using FRET (37).

The principle difference between the conformation of apoA-I on the two different sized diameter rHDL particles is that fewer intramolecular cross-links and more intermolecular cross-links were identified within the $80 \AA$ apoA-I particles compared to $96 \AA$ particles. The conformation obtained for 2 molecules of apoA-I on the smaller $80 \AA$ diameter rHDL particles is similar to that proposed for the $96 \AA$ diameter rHDL particles, and is shown in Figure 5, Panels A, B and C. More specifically, shown in Figure 5, Panel D, the pair of intermolecular cross-links (red lines) that were common to both sized rHDL particles, Lys 118 to Lys 140 and $\mathrm{Lys}_{40}$ and $\mathrm{Lys}_{239}$ suggest that the middle of the two apoA-I molecules have similar orientations and that the N-terminal, Lys 40 , and C-terminal, $\mathrm{Lys}_{239}$, ends are positioned close together. However, the latter pair of Lys residues appear to have apparently slid along the apoA-I chain so that they now lie closer to Lys94. The cross-link between $\alpha-\mathrm{NH}_{2}$ and $\mathrm{Lys}_{118}$ present in the $80 \AA$ diameter particle is consistent with this assertion. Thus, from these data, it appears that the extent of apoA-I to apoA-I protein-protein interactions provides needed stability to the lipid-bound conformation as well as allowing accommodation of phospholipid molecules. Perhaps the degree to which the $\mathrm{N}$ - and C-termini can form stabilizing interactions may indirectly determine the amount of phospholipid packaged within an rHDL particle.

\section{DISCUSSION}

In these studies, the experimentally derived molecular constraints obtained from CCL/MS have been used to refine a molecular model of lipid-bound apoA-I on two different diameter phospholipid containing particles. Careful examination of cross-links that did not fit the expected LL5/5 conformation suggested by earlier studies on lipid-bound apoA-I conformation, mainly involve the $\mathrm{N}$ - and C-terminal end of 2 antiparallel lipid-bound apoA-I molecules. In our previous studies, we hypothesized that lipid-bound apoA-I wrapped around the phospholipid bilayer like a "belt-buckle" with a N-terminal "hairpin" fold making contact with the C-terminus on $96 \AA$ rHDL complexes (32). In these current studies, we have now further refined and validated our model by using both longer and shorter arm length crosslinkers and find that these new data fit the previously proposed "belt-buckle" for apoA-I on 96 $\AA$ rHDL complexes (Figure 4). This exhaustive study also provides direct experimental evidence for a tighter "buckle" of apoA-I on $80 \AA$ rHDL complexes wherein the N- and Ctermini of apoA-I fold back and stabilize a smaller particle diameter, thus enabling particle expansion as more phospholipid and cholesterol can be accommodated (Figure 5). This conclusion was most evident from the presence of N-terminal cross-links at the alpha amino terminus-K118, K94-K239 and K40-K239 verified by MS/MS sequencing.

CCL/MS was first used to investigate the lipid-bound structure of apoA-I in 2003 by Davidson and Hilliard (31). From these studies they proposed three possible orientations for the arrangement of 2 molecules of apoA-I along the edge of a recombinant rHDL; antiparallel, slid-antiparallel where the N-termini are offset, and an antiparallel Z-conformation in which the two molecules of apoA-I crossed one another. In 2005, a subsequent study by Silva et al. (33) utilizing MS/MS sequencing the Z-belt conformation was shown to be unverifiable. In this same study, the authors concluded that lipid-bound apoA-I on 96 and $80 \AA$ particles were similar in conforming to both LL5/5 and LL5/2 conformations, but differing in that the central or hinge region (helices 5-7) may extend further away from the particle on the smaller $80 \AA$ 
particles $(29,33)$. This hinge or "looped belt" region has also been hypothesized to account for heterogeneity in DMPC rHDL reconstituted particles using a combination of EPR and FRET (44) as well as reconstitution experiments of truncated mutant forms of apoA-I with phospholipids (13). However, in a recent study employing molecular dynamic simulations of POPC particle reduction, the predicted detachment of the hinged domain from the edge of the rHDL particle was not observed (44). Instead, in these studies, the "belt" remained attached to the particles' edge, although there was significant conformational change in apoA-I as the protein conformed to the particles' smaller diameter. In these studies, molecular dynamic simulations showed that lipid-bound apoA-I wrapped in a belt conformation on $96 \AA$ particles, but reduction in the amount of phospholipid in the particle resulted in a "saddle-shaped" structure. In this model, the conformation of two molecules of lipid-bound apoA-I twisted to conform to the minimal surface edge of the lipid bilayer as the particle assumed a progressively smaller diameter (45).

Studies utilizing limited proteolysis of lipid-bound wild-type (46) and A173C apo A-I (47) suggest that the $\mathrm{N}$ - and $\mathrm{C}$-terminal regions are more highly susceptible to cleavage on recombinant POPC complexes. Protease cleavage sites suggest that these regions of lipidbound apoA-I are more "accessible" and thus, less likely to be buried in phospholipid. In addition to providing new information concerning the conformation of the $\mathrm{N}$ - and $\mathrm{C}$-terminal ends of lipid-bound apoA-I, these studies also suggest that the central domain of apoA-I is essential in providing a large portion of its kinetic stability. SDS-resistance observed in both Q109C and Q132C apoA-I homo- and heterodimers suggest that the conformation of helix 4 and 5 play a pivotal role in global apoA-I structure. Interestingly, inter-molecular cross-links between two molecules of wild-type apoA-I are abundantly found in this same domain (32, 33). Possibly the occurrence of salt-bridge formation in this region explains its significant stability and frequency of interaction $(28,34)$.

In conclusion, the use of CCL/MS has provided information on which to build a model of 2 lipid-bound apoA-I molecules on 96 and $80 \AA$ POPC recombinant particles. These models show that the $\mathrm{N}$ - and $\mathrm{C}$-terminal regions of the proteins interact with each other to an extent depending on the amount of lipid contained in the particle. These models of lipid-bound apoAI should be interpreted with care since they are derived from apoA-I that has been "unfolded" with sodium cholate prior to its formation of discrete phospholipid containing particles. Further studies are needed to determine the conformation of 2 apoA-I molecules containing phospholipid particles generated from the cholesterol and phospholipid transporter, ABCA1.

\section{Supplementary Material}

Refer to Web version on PubMed Central for supplementary material.

\section{Acknowledgements}

These studies were supported by grants from the National Institutes of Health NHLBI HL-49373 and HL-64163 (MST).

\section{REFERENCES}

1. Rader DJ. Molecular regulation of HDL metabolism and function: implications for novel therapies. J Clin Invest 2006;116:3090-3100. [PubMed: 17143322]

2. Linsel-Nitschke P, Tall AR. HDL as a target in the treatment of atherosclerotic cardiovascular disease. Nat Rev Drug Discov 2005;4:193-205. [PubMed: 15738977]

3. Oram JF, Heinecke JW. ATP-binding cassette transporter A1: a cell cholesterol exporter that protects against cardiovascular disease. Physiol Rev 2005;85:1343-1372. [PubMed: 16183915]

4. Pownall HJ, Ehnholm C. The unique role of apolipoprotein A-I in HDL remodeling and metabolism. Curr Opin Lipidol 2006;17:209-213. [PubMed: 16680023] 
5. Saito H, et al. Domain structure and lipid interaction in human apolipoproteins A-I and E, a general model. J. Biol. Chem 2003;278:23227-23232. [PubMed: 12709430]

6. Timmins JM, et al. Targeted inactivation of hepatic Abcal causes profound hypoalphalipoproteinemia and kidney hypercatabolism of apoA-I. J Clin Invest 2005;115:1333-1342. [PubMed: 15841208]

7. Zannis VI, Chroni A, Krieger M. Role of apoA-I, ABCA1, LCAT, and SR-BI in the biogenesis of HDL. J Mol Med 2006;84:276-294. [PubMed: 16501936]

8. von Eckardstein A. Differential diagnosis of familial high density lipoprotein deficiency syndromes. Atherosclerosis 2006;186:231-239. [PubMed: 16343506]

9. Sorci-Thomas MG, Thomas MJ. The effects of altered apolipoprotein A-I structure on plasima HDL concentration. Trends Cardiovasc. Med 2002;12:121-128. [PubMed: 12007737]

10. Thuahnai ST, et al. Scavenger receptor class B type I-mediated cholesteryl ester-selective uptake and efflux of unesterified cholesterol. Influence of high density lipoprotein size and structure. J Biol Chem 2004;279:12448-12455. [PubMed: 14718538]

11. Connelly MA, Williams DL. SR-BI and HDL cholesteryl ester metabolism. Endocr Res 2004;30:697703. [PubMed: 15666814]

12. Segrest JP, et al. The amphipathic helix in the exchangeable apolipoproteins: a review of secondary structure and function. J.Lipid Res 1992;33:141-166. [PubMed: 1569369]

13. Li L, et al. Double belt structure of discoidal high density lipoproteins: molecular basis for size heterogeneity. J Mol Biol 2004;343:1293-1311. [PubMed: 15491614]

14. Borhani DW, et al. Crystal structure of truncated human apolipoprotein A-I suggests a lipid-bound conformation. Proc.Natl.Acad.Sci.USA 1997;94:12291-12296. [PubMed: 9356442]

15. Palgunachari MN, et al. Only the two end helixes of eight tandem amphipathic helical domains of human apo A-I have significant lipid affinity - Implications for HDL assembly. Arterioscler.Thromb.Vasc.Biol 1996;16:328-338. [PubMed: 8620350]

16. Zhu HL, Atkinson D. Conformation and lipid binding of a C-terminal (198-243) Peptide of human apolipoprotein a-I. Biochemistry 2007;46:1624-1634. [PubMed: 17279626]

17. Zhu HL, Atkinson D. Conformation and lipid binding of the N-terminal (1-44) domain of human apolipoprotein A-I. Biochemistry 2004;43:13156-13164. [PubMed: 15476409]

18. Beckstead JA, et al. Combined N- and C-Terminal Truncation of Human Apolipoprotein A-I Yields a Folded, Functional Central Domain. Biochemistry 2005;44:4591-4599. [PubMed: 15766290]

19. Lagerstedt JO, et al. EPR spectroscopy of site-directed spin labels reveals the structural heterogeneity in the N-terminal domain of apo-AI in solution. J Biol Chem. 2007

20. Oda MN, et al. The C-terminal domain of apolipoprotein A-I contains a lipid-sensitive conformational trigger. Nat Struct Biol 2003;10:455-460. [PubMed: 12754494]

21. Saito $\mathrm{H}$, et al. Alpha-helix formation is required for high affinity binding of human apolipoprotein A-I to lipids. J Biol Chem 2004;279:20974-20981. [PubMed: 15020600]

22. Fang YL, Gursky O, Atkinson D. Lipid-binding studies of human apolipoprotein A-I and its terminally truncated mutants. Biochemistry 2003;42:13260-13268. [PubMed: 14609337]

23. Fang Y, Gursky O, Atkinson D. Structural studies of N- and C-terminally truncated human apolipoprotein A-I. Biochemistry 2003;42:6881-6890. [PubMed: 12779343]

24. Tanaka M, et al. Contributions of the $\mathrm{N}$ - and C-terminal helical segments to the lipid-free structure and lipid interaction of apolipoprotein A-I. Biochemistry 2006;45:10351-10358. [PubMed: 16922511]

25. Scott BR, et al. The N-terminal globular domain and the first class A amphipathic helix of apolipoprotein A-I are important for lecithin:cholesterol acyltransferase activation and the maturation of high denisty lipoprotein in vivo. J. Biol. Chem 2001;276:48716-48724. [PubMed: 11602583]

26. Chroni A, et al. The central helices of ApoA-I can promote ATP-binding cassette transporter A1 (ABCA1)-mediated lipid efflux. Amino acid residues 220-231 of the wild-type ApoA-I are required for lipid efflux in vitro and high density lipoprotein formation in vivo. J Biol Chem 2003;278:67196730. [PubMed: 12488454]

27. Ajees AA, et al. Crystal structure of human apolipoprotein A-I: Insights into its protective effect against cardiovascular diseases. Proc Natl Acad Sci U S A. 2006 
28. Klon AE, Segrest JP, Harvey SC. Comparative models for human apolipoprotein A-I bound to lipid in discoidal high-density lipoprotein particles. Biochemistry 2002;41:10895-10905. [PubMed: 12206659]

29. Davidson WS, Silva RA. Apolipoprotein structural organization in high density lipoproteins: belts, bundles, hinges and hairpins. Curr Opin Lipidol 2005;16:295-300. [PubMed: 15891390]

30. Young MM, et al. High throughput protein fold identification by using experimental constraints derived from intramolecular cross-links and mass spectrometry. Proc Natl Acad Sci U S A 2000;97:5802-5806. [PubMed: 10811876]

31. Davidson WS, Hilliard GM. The spatial organization of apolipoprotein A-I on the edge of discoidal high density lipoprotein particles - A mass spectrometry study. J. Biol. Chem 2003;278:27199_ 27207. [PubMed: 12724319]

32. Bhat $\mathrm{S}$, et al. Intermolecular contact between globular $\mathrm{N}$-terminal fold and $\mathrm{C}$-terminal domain of ApoA-I stabilizes its lipid-bound conformation: studies employing chemical cross-linking and mass spectrometry. J Biol Chem 2005;280:33015-33025. [PubMed: 15972827]

33. Silva RA, et al. A mass spectrometric determination of the conformation of dimeric apolipoprotein A-I in discoidal high density lipoproteins. Biochemistry 2005;44:8600-8607. [PubMed: 15952766]

34. Segrest JP, et al. Structure and function of apolipoprotein A-I and high-density lipoprotein. Curr Opin Lipid 2000;11:105-115.

35. Maiorano JN, et al. Identification and structural ramifications of a hinge domain in apolipoprotein A-I discoidal high-density lipoproteins of different size. Biochemistry 2004;43:11717-11726. [PubMed: 15362856]

36. Nichols AV, et al. Effects of guanidine hydrochloride on human plasma high density lipoproteins. Biochim Biophys Acta 1976;446:226-239. [PubMed: 184833]

37. Li HH, et al. Structural determination of lipid-bound ApoA-I using fluorescence resonance energy transfer. J. Biol. Chem 2000;275:37048-37054. [PubMed: 10956648]

38. Alexander ET, et al. Apolipoprotein A-I helix 6 negatively charged residues attenuate lecithin:cholesterol acyltransferase (LCAT) reactivity. Biochemistry 2005;44:5409-5419. [PubMed: 15807534]

39. Sorci-Thomas MG, et al. High level secretion of wild-type and mutant forms of human proapoA-I using baculovirus-mediated Sf-9 cell expression. J.Lipid Res 1996;37:673-683. [PubMed: 8728328]

40. MacFarlane AS. Efficient trace-labeling of proteins with iodine. Nature 1958;182:53.

41. Zhu ZB, Atkinson TP, Volanakis JE. A novel type II complement C2 deficiency allele in an AfricanAmerican family. J Immunol 1998;161:578-584. [PubMed: 9670930]

42. Reynolds JA, Tanford C. The gross conformation of protein-sodium dodecyl sulfate complexes. J Biol Chem 1970;245:5161-5165. [PubMed: 5528242]

43. Manning M, Colon W. Structural basis of protein kinetic stability: resistance to sodium dodecyl sulfate suggests a central role for rigidity and a bias toward beta-sheet structure. Biochemistry 2004;43:11248-11254. [PubMed: 15366934]

44. Martin DD, et al. Apolipoprotein A-I assumes a "looped belt " conformation on reconstituted high density lipoprotein. J Biol Chem 2006;281:20418-20426. [PubMed: 16698792]

45. Catte A, et al. Novel changes in discoidal high density lipoprotein morphology: a molecular dynamics study. Biophys J 2006;90:4345-4360. [PubMed: 16581834]

46. Roberts LM, et al. Structural analysis of apolipoprotein A-I: Limited proteolysis of methioninereduced and -oxidized lipid-free and lipid-bound human apo A-I. Biochemistry 1997;36:7615-7624. [PubMed: 9200714]

47. Calabresi L, et al. Limited proteolysis of a disulfide-linked apoA-I dimer in reconstituted HDL. J Lipid Res 2001;42:935-942. [PubMed: 11369801]

\section{The abbreviations used are}

apoA-I, apolipoprotein A-I; HDL, high density lipoprotein; rHDL, recombinant HDL; POPC, 1-palmitoyl-2-oleoyl phosphotidylcholine; DSG, disuccinimidylglutarate; DSP, dithiobis (succinimidylpropionate); EGS, ethylene glycolbis(succinimidylsuccinate); QTOF MS, 
quadrupole time of flight mass spectrometry; MS/MS, tandem mass spectrometry; LC, liquid chromatography. 


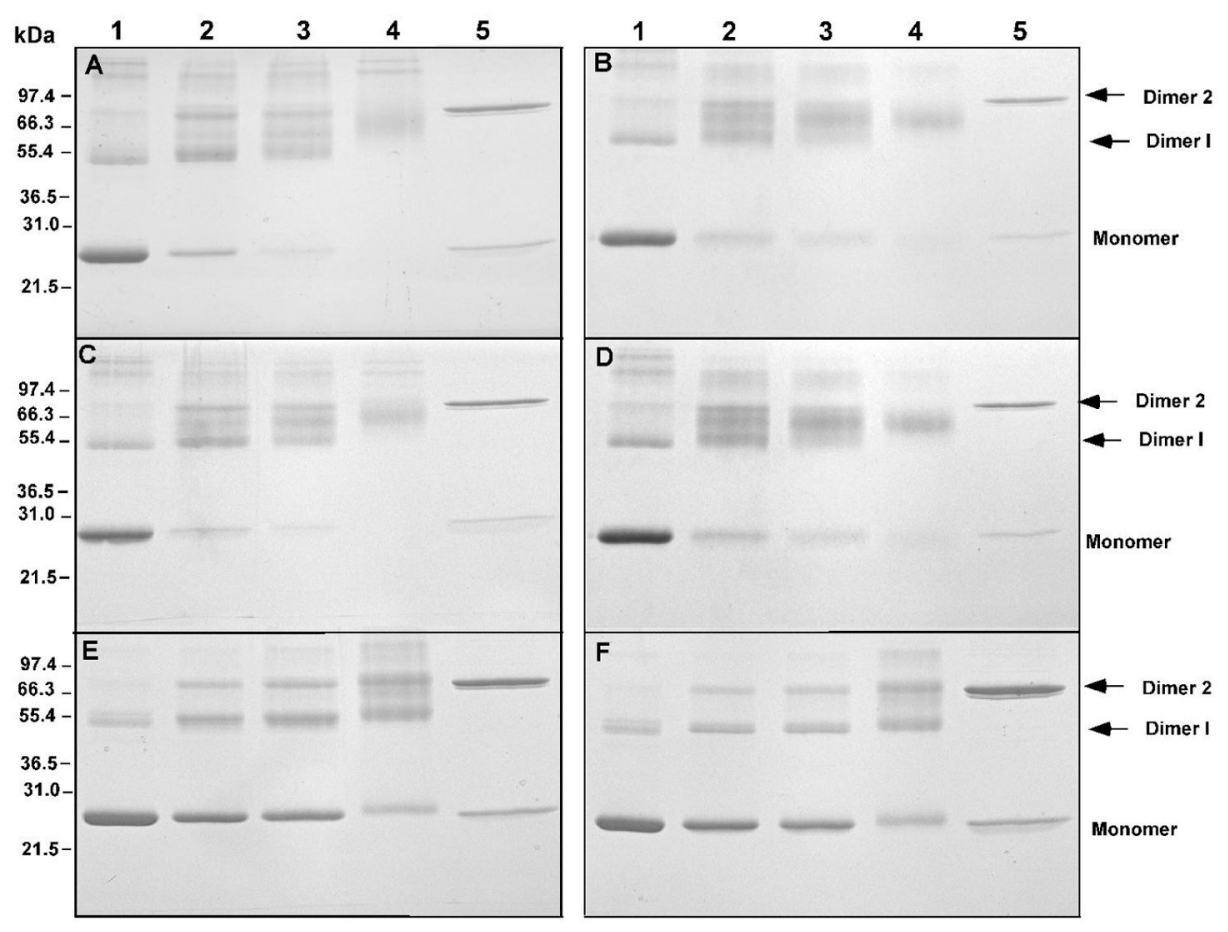

FIGURE 1. Non-Reducing $12 \%$ SDS-PAGE of Cross-linked POPC rHDL containing apoA-I Cross-linked products from 96 (Panels A, C, and E) and $80 \AA$ diameter (Panels B, D, and F) POPC rHDL cross-linked with DSG (Panels A and B), DSP (Panels C and D) and EGS (Panels $\mathrm{E}$ and $\mathrm{F}$ ) at fixed molar ratios of cross-linker to protein for $5 \mathrm{~min}$ at $37^{\circ} \mathrm{C}$, as described under "Materials and Methods". All cross-linked samples were separated by $12 \%$ non-reducing SDSPAGE and visualized using Simply Blue ${ }^{\mathrm{TM}}$. For Panels A-F, Lane 1, lipid-free apoA-I treated at a molar ratio of $5: 1$ cross-linker to apoA-I ; Lane 2, lipid-bound apoA-I treated at a molar ratio of $10: 1$ cross-linker to apoA-I; Lane 3, lipid-bound apoA-I treated at a molar ratio of $20: 1$ cross-linker to apoA-I; Lane 4, lipid-bound apoA-I treated at a molar ratio of $200: 1$ cross-linker to apoA-I; Lane 5, unreduced lipid-free Q109C apoA-I homodimer. 
A.

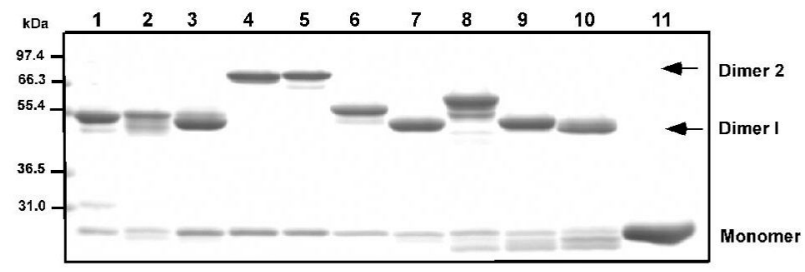

B.

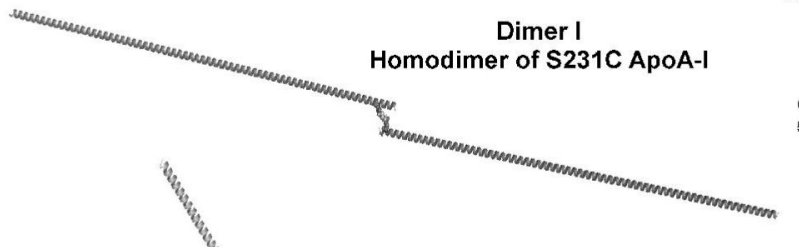

C.
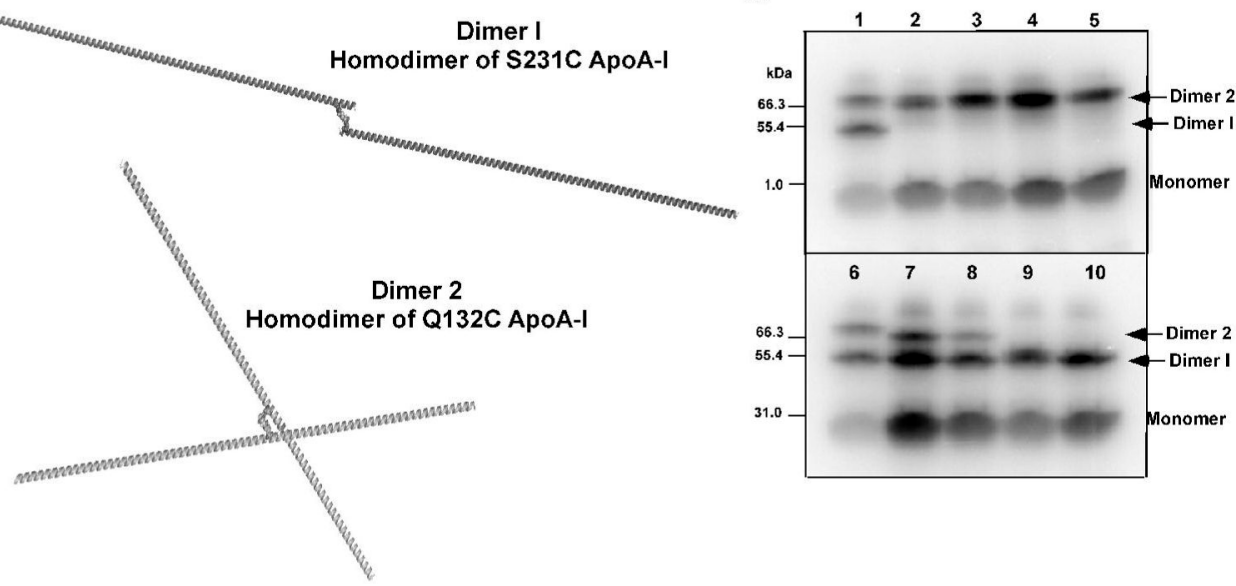

FIGURE 2. Electrophoretic Migration of Homo- and Hetero-Dimers of Cysteine-Containing ApoA-I

Panel A shows the altered molecular weight migration of cysteine-containing apoA-I

homodimers. Lanes 1-11 show the electrophoretic migration of lipid-free cysteine-containing apoA-I homodimers separated by non-reducing 12\% SDS-PAGE. Lane 1, Q5C apoA-I; Lane 2, S55C apoA-I; Lane 3, Q84C apoA-I; Lane 4, Q109C apoA-I; Lane 5, Q132C apoA-I; Lane 6, A154C apoA-I; Lane 7, A176C apoA-I; Lane 8, A194C apoA-I; Lane 9, Q216C apoA-I; Lane 10, S231C apoA-I; and Lane 11, wild-type apoA-I monomer. Cysteine containing apoAI monomers can spontaneously oxidize to form homodimers in the absence of reducing agents. All apoA-I mutants shown in panel A were subjected to mass spectrometry to confirm that their experimental molecular weight matched its theoretical molecular weight. Interestingly, Q109C, Q132C show the largest deviation from the expected 56,000 Da molecular migration on SDS PAGE for an apoA-I dimer. Deviations from expected electrophoretic migration may result from the inability of SDS to linearize these dimers. Panel B, shows a hypothetical model illustrating the conformation for a homodimer of S231C apoA-I, referred to as Dimer 1, and a homodimer of Q132C apoA-I, referred to as Dimer 2. The SDS PAGE migration may be altered in the case of D2 as a result of incomplete unfolding, linearization and interference in migrating through the polyacrylamide pores, resulting in a larger than actual molecular size. Studies were conducted to determine the migration of cysteine-containing apoA-I heterodimers as described in "Materials and Methods". Panel C, Lanes 1 and 6 show the SDS PAGE migration of the mixture of radiolabeled homodimers (Dimer $1+$ Dimer 2 ) containing ${ }^{125}$ I-labeled Q132C $+{ }^{125}$ I-labeled S231C apoA-I $\left(1 \times 10^{5} \mathrm{cpm}\right.$ each $)$; Lanes $2-5$, show the results of reductionoxidation for mixtures of ${ }^{125} \mathrm{I}-\mathrm{Q} 132 \mathrm{C}$ and unlabeled cysteine containing apoA-I mutants. Lane 2, ${ }^{125} \mathrm{I}-\mathrm{Q} 132 \mathrm{C}+$ unlabeled Q84C apoA-I; Lane 3, ${ }^{125} \mathrm{I}-\mathrm{Q} 132 \mathrm{C}+$ unlabeled Q109C apoA-I; Lane 4, ${ }^{125} \mathrm{I}$ Q132C + unlabeled Q132C apoA-I; Lane 5, ${ }^{125} \mathrm{I}-\mathrm{Q} 132 \mathrm{C}+$ unlabeled A176C apoAI. Lanes 7-10 show the results of reduction-oxidation for mixtures of ${ }^{125} \mathrm{I}$-labeled S231C and unlabeled cysteine containing apoA-I mutants. Lane 7, ${ }^{125} \mathrm{I}-\mathrm{S} 231 \mathrm{C}+$ unlabeled Q109C apoA- 
I; Lane 8, ${ }^{125} \mathrm{I}-\mathrm{S} 231 \mathrm{C}+$ unlabeled Q132C apoA-I; Lane 9, ${ }^{125} \mathrm{I}^{-} \mathrm{S} 231 \mathrm{C}+$ unlabeled A176C apoA-I; Lane 10, ${ }^{125} \mathrm{I}^{-} \mathrm{S} 231 \mathrm{C}+$ unlabeled Q84C apoA-I. The migration of heterodimers containing radiolabeled Q132C apoA-I and all combinations of cysteine containing apoA-I mutants as the Dimer 2 suggest that a single covalent attachment within the central domain of apoA-I retards SDS PAGE migration, while heterodimers containing radiolabeled S231C apoA-I showed a mixture of both Dimer 1 and Dimer 2. 
A

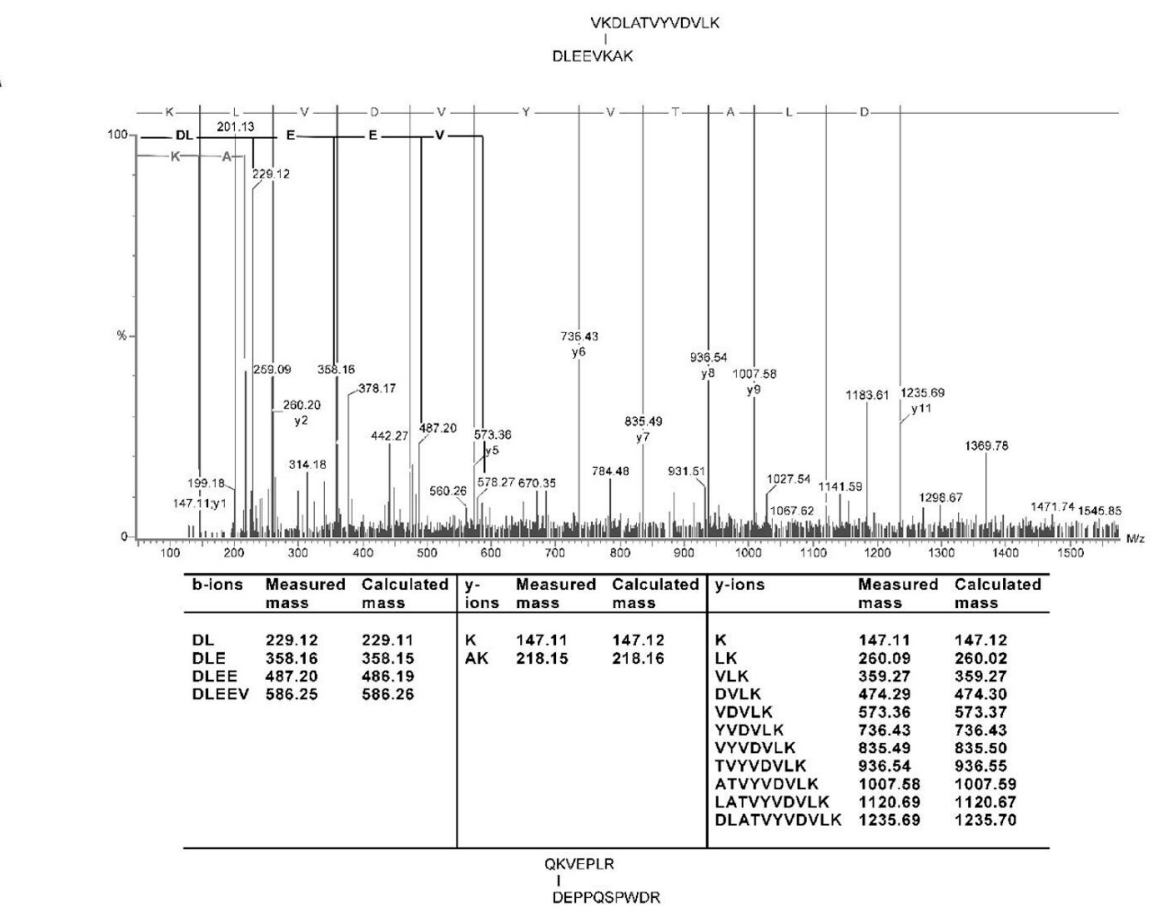

B

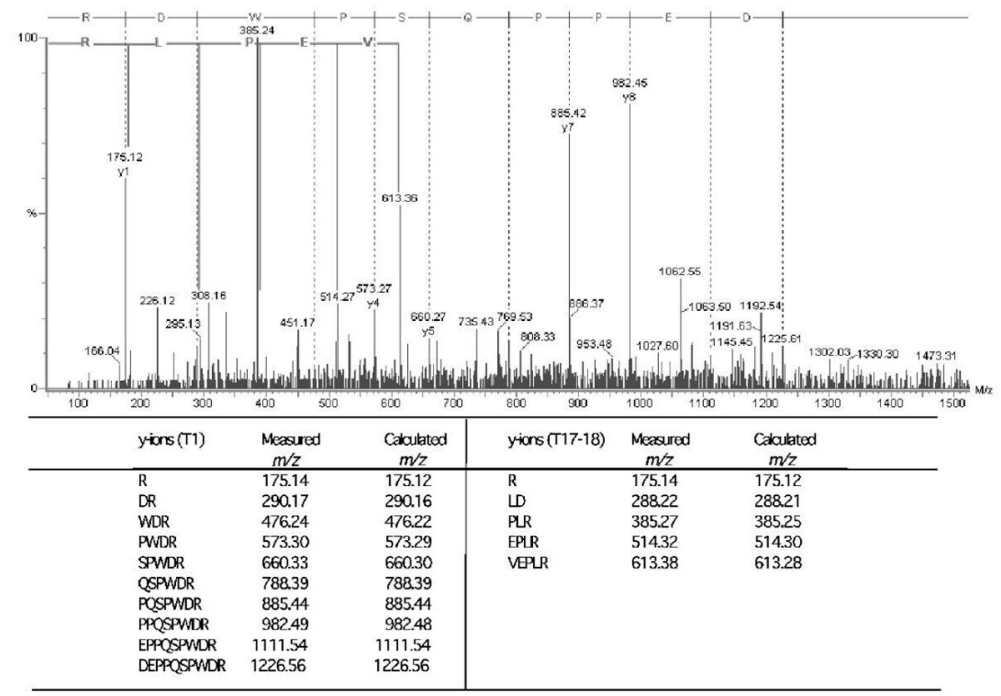

FIGURE 3. QTOF-MS/MS Spectrum of Intermolecular Cross-links from DSP treated ApoA-I on rHDL

Panel A, shows an intermolecular cross-link on $96 \AA$ AHDL at Lysines 12 and 94 from which $96 \AA$ rHDL was cross-linked with DSG and in-gel trypsin digest, peptide extraction and sequencing was carried out as described under "Materials and Methods". The top section of panel A shows the product ion spectrum for the 2488.2725 Da fragment from the Dimer 1 product. The MS/MS spectrum was obtained from the triply charged ion $m / z 830.43$ and the sequence of the peptide is shown as single letter abbreviations. A y series for KA and KLVDVYVTALD and $a \mathrm{~b}$ series for DLEEV identified the peptide fragments. The lower section of panel A shows $\mathrm{m} / \mathrm{z}$ for the experimental and theoretical product ions. Panel B, shows an intermolecular cross-linked apoA-I on $80 \AA$ at lysines $\alpha$-NH2 and 118. The $80 \AA$ rHDL was 
cross-linked with DSG and in-gel trypsin digest was carried out as described under "Materials and Methods". The top section of panel B shows the product ion spectrum for the 2190.0742 Da fragment from the Dimer 1 product. The MS/MS spectrum was obtained from the triply charged ion $m / z 731.01$ and the sequence of the peptide is shown as single letter abbreviations. Y-series for DEPPQSPWDR and VEPLR identified the peptide fragment. The lower section of panel B shows $\mathrm{m} / \mathrm{z}$ for the experimental and theoretical product ions. 


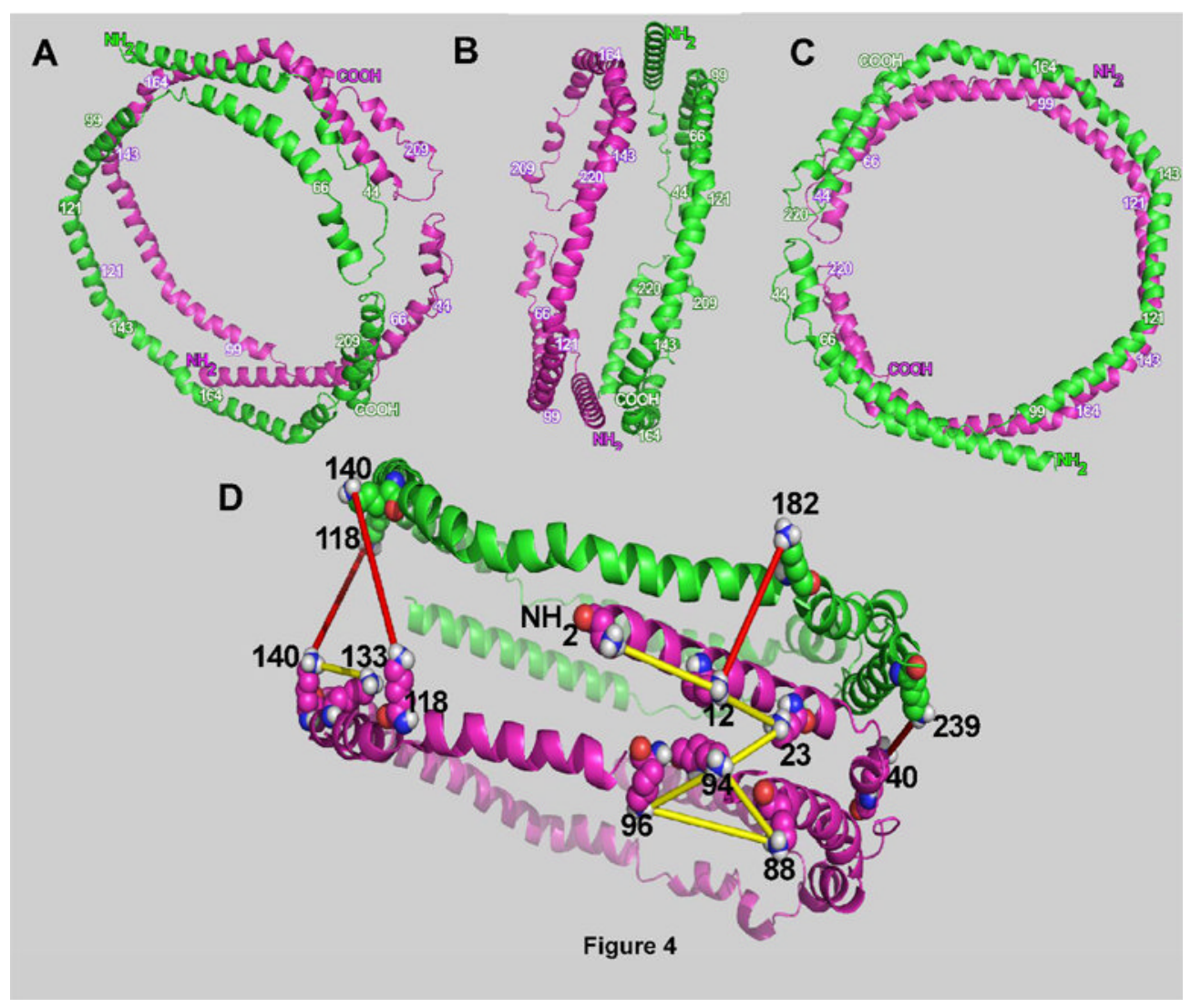

FIGURE 4. Model of the Lipid-bound Conformation for 2 Molecules of ApoA-I on a 96А̊ POPC rHDL Particle

MS/MS sequenced DSP cross-links as listed in Table 1 were used as molecular constraints for modeling the lipid-bound conformation of 2 molecules of apoA-I. Different colors indicate individual apoA-I molecules with the corresponding colored amino acid residue number. Red lines indicate intermolecular cross-links, while yellow lines indicate intramolecular crosslinks. 


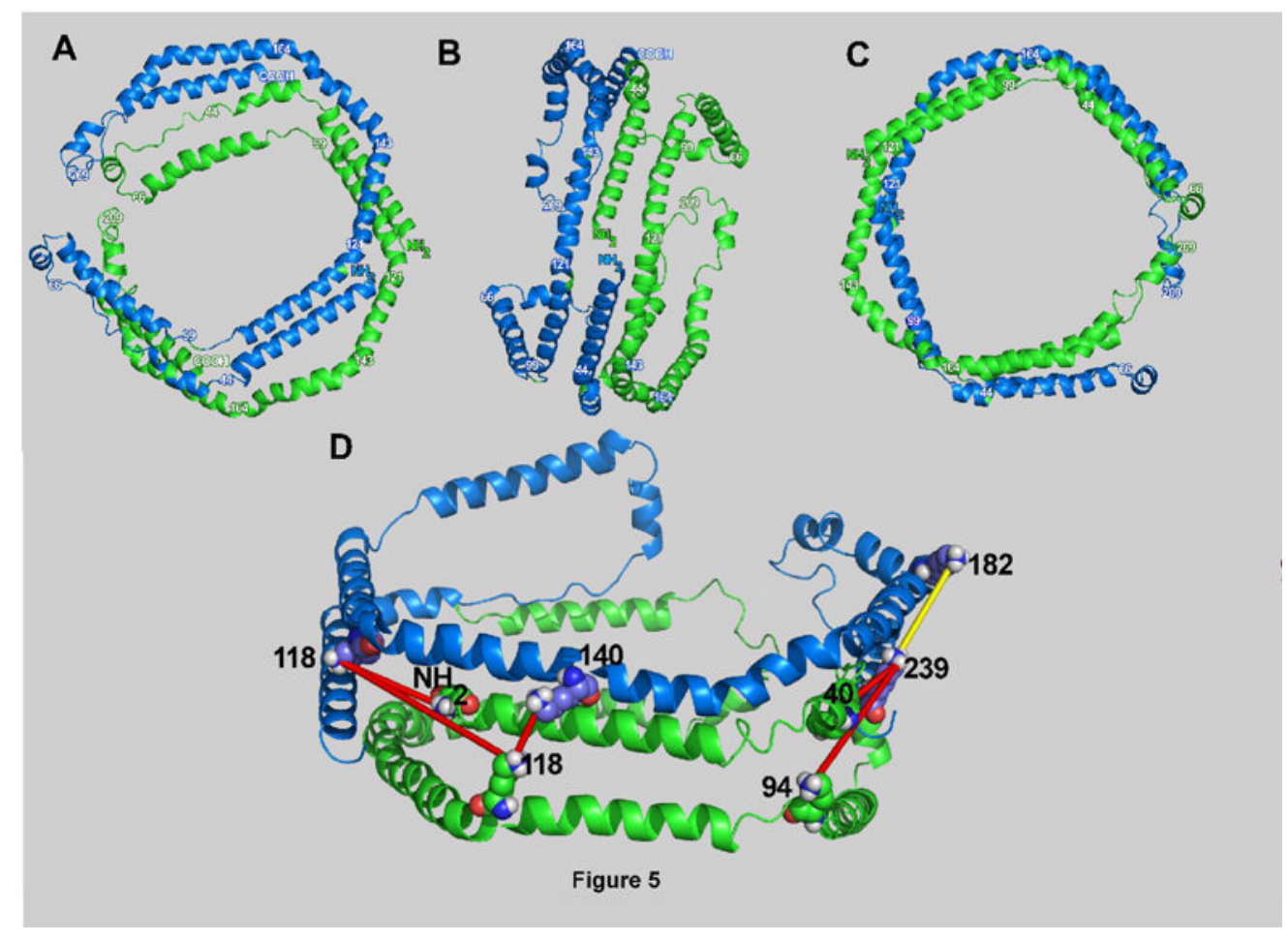

FIGURE 5. Model of the Lipid-bound Conformation for 2 Molecules of ApoA-I on a 80A POPC rHDL Particle

MS/MS sequenced DSP cross-links as listed in Table 2 were used as molecular constraints for modeling the lipid-bound conformation of 2 molecules of apoA-I bound to lipid. Different colors indicate individual apoA-I molecules with their corresponding colored animo acid residue number. Red lines indicate intermolecular cross-links, while yellow lines indicate intramolecular cross-links. 


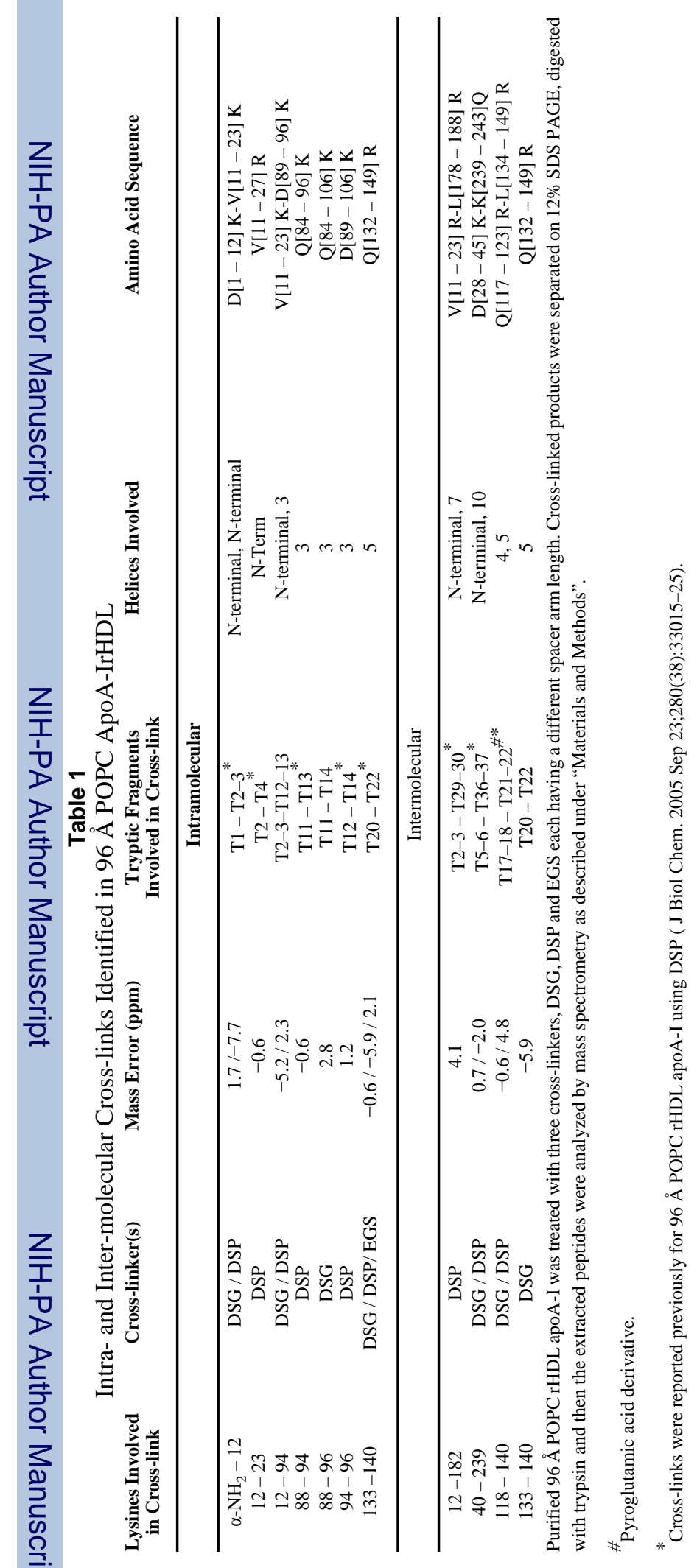




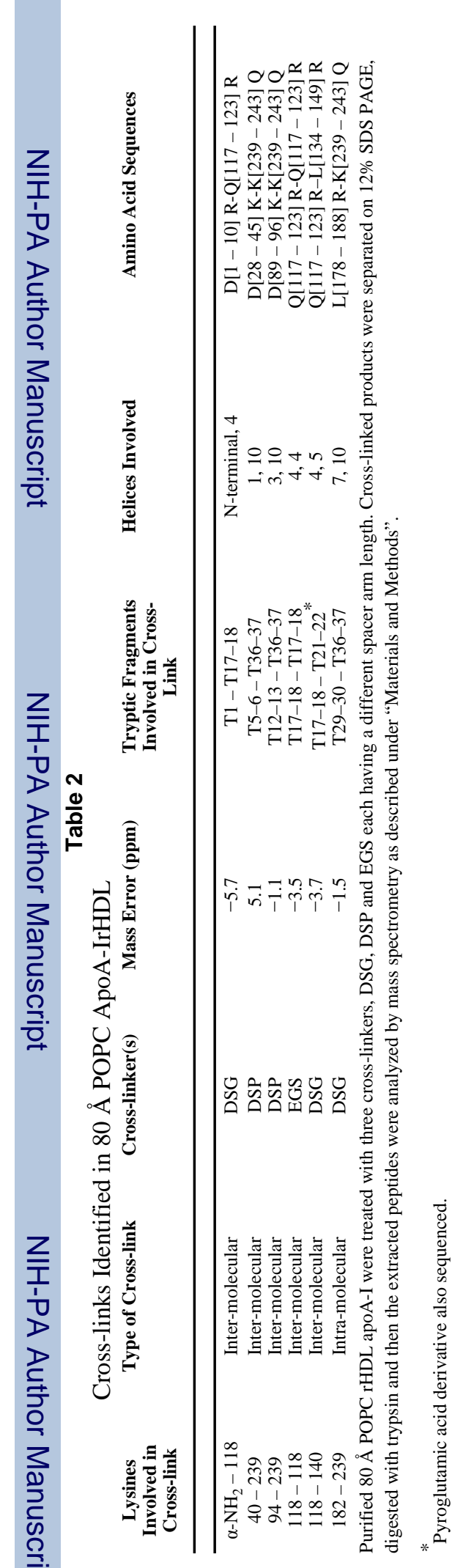

Biochemistry. Author manuscript; available in PMC 2008 September 25. 